\title{
Development of Intelligent Public Trash Can Based on Machine Vision and Learning
}

\author{
Longyu Gao ${ }^{1}$, Fengzhi Dai ${ }^{1,2}{ }^{*}$, Zhiqing Xiao ${ }^{1}$, Jiangyu Wu ${ }^{1}$, Zilong Liu ${ }^{1}$ \\ ${ }^{1}$ Tianjin University of Science and Technology, China; \\ 2 Tianjin Tianke Intelligent and Manufacture Technology CO., LTD, China; \\ E-mail:*daifz@tust.edu.cn \\ www.tust.edu.ch
}

\begin{abstract}
At present, with the maturity of machine vision technology and the continuous expansion of application fields, there have been many intelligent trash cans based on machine vision, which can realize certain garbage identification and automatic classification. However, due to certain technical limitations of machine vision, it is impossible to identify all garbage. In this paper, a smart public trash can based on machine vision and auxiliary sensors is proposed. In addition to realizing machine vision to identify and automatically classify garbage, sensors will also be used to assist in identifying garbage to solve problems such as the same garbage classification of different shapes. At the same time, enhanced learning will be added to realize the self-learning of the trash can, so as to achieve the goal of continuously increasing identifiable types.
\end{abstract}

Keywords: machine vision, machine learning, garbage classification, auxiliary sensors

\section{Introduction}

With the development of society and the increasing improvement of people's living standards, society is paying more and more attention to the problem of garbage classification, and various regions have also implemented relevant garbage classification policies. However, due to various types of garbage and people's poor awareness of autonomous classification, the classification effect is difficult to achieve the expected effect. And as a result, intelligent sorting trash bins based on various technologies have emerged.

Most the intelligent sorting trash can lies in automatically identifying the type of trash. At present, with the maturity of machine vision technology and a wide range of applications, an intelligent classification of trash cans based on this technology has been born, which can realize certain garbage identification and automatic classification. However, due to certain technical limitations of machine vision, it is impossible to recognize all garbage. For example, it is difficult to recognize the same garbage under different shapes.

In summary, the smart trash can not only needs to be based on machine vision, but also needs to be supplemented when machine recognition cannot be effective. Therefore, a smart public trash can based on machine vision and auxiliary sensors is proposed. In addition to realizing to recognize garbage and automatically classify it by machine vision, sensors will also be installed to assist in identifying garbage to solve problems such as the same garbage classification of different shapes. At the same time, enhanced learning will be added to realize the self-learning of the trash can, so as to achieve the goal of continuously increasing identifiable types.

\section{Overall Design Scheme of the System}

According to actual application scenarios and market requirements, combined with machine vision, sensor detection technology, and wireless communication 
technology, this paper designs a smart public trash can based on machine vision to realize automatic garbage sorting and machine learning (continuously increasing the number of identifiable types), the classification of different states of the first class of garbage and the real-time monitoring of garbage filling.

When the smart trash can detects that the rubbish is input, the trash can uses a visual camera to capture and identify the dropped rubbish ${ }^{1}$. Then compare and analyze the data in the database to determine the type of garbage, and then use its own mechanical structure to sort it into the corresponding garbage bin.

For the unrecognizable garbage, the trash can will work through the interaction of metal sensors, humidity sensors, VOC odor sensors and capacitive sensors to sort the garbage into the corresponding garbage cans, mainly to solve the problem of garbage occupying the identification position. At the same time, the unrecognizable garbage is photographed, uploaded to the server, and re-trained to determine the type of garbage. When the garbage is recognized again next time, the garbage classification will be performed directly through machine vision. At the same time, each trash can will share data, which can greatly improve the learning speed of the trash can and shorten the learning cycle. The flow chart of the intelligent public trash can based on machine vision is shown in Fig.1.

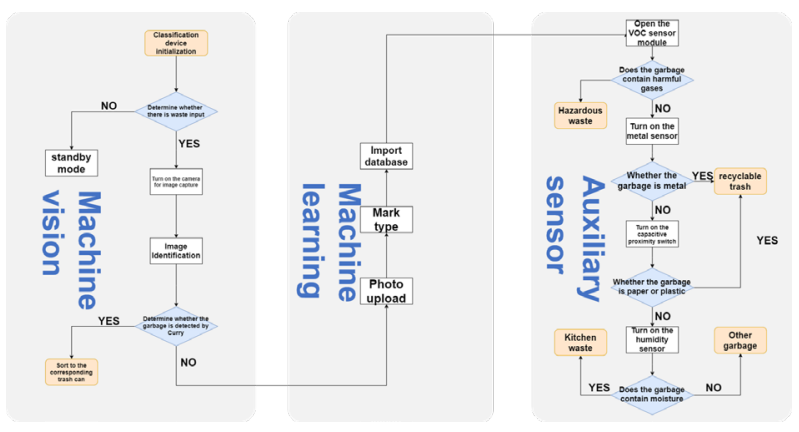

Fig.1. The Overall flow chart of intelligent public trash bin based on machine vision

\section{Design of Hardware Control System}

This system selects STM32F103 single-chip microcomputer as the core controller, and combines OpenMV and peripheral modules to realize the control system function. It mainly include: STM32, OpenMV, metal sensor, humidity sensor, VOC smell sensor and capacitance sensor, communication module, motor, etc. The hardware control part of the system is introduced below.

\subsection{Main control chip}

STM32f407zet6 is selected as the core controller of the hardware control system, which is mainly used to realize the functions of data acquisition and analysis and execution of component motion control ${ }^{2}$.

The processor of STM32f407zet6 is Arm Cortex-M4 high-performance core, the maximum operating frequency is $168 \mathrm{MHz}$, and the package form is $1 \mathrm{qfp} 144$. Its peripheral configuration is powerful, supporting communication interfaces such as SP interface, I2C interface, USB interface, USART interface, and peripherals such as ADC and timer. STM32f407zet6 is shown in Fig.2.

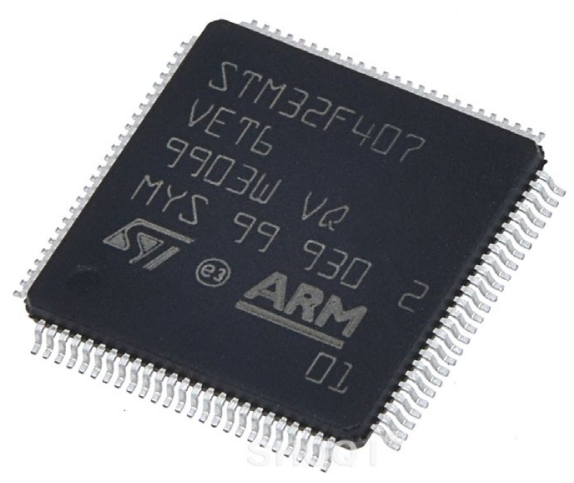

Fig.2. Main control chip STM32f407zet6

\subsection{Metal sensor}

The function of material recognition is realized through the metal sensor module. The system uses metal sensors to identify metals. This system uses LJ18A3-8-Z/BX type metal sensor. The sensor has the advantages of fast response speed, strong anti-interference ability, waterproof and corrosion resistance, and very stable performance.

It is widely used in automated assembly lines, intelligent hardware and security systems. The operating voltage of the sensor is $6-36 \mathrm{~V}$ (DC), and the switch contains a high-frequency oscillation circuit, a detection circuit, an amplifying circuit, a de-transmitting circuit and an output circuit. The metal sensor is shown in Fig.3. 


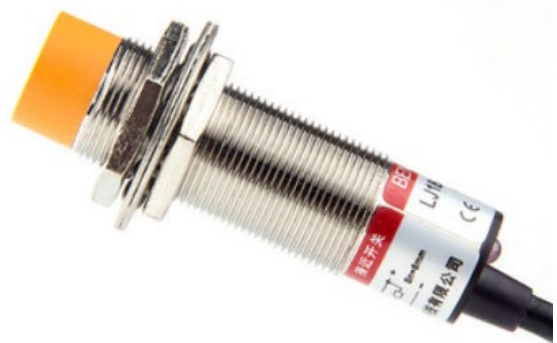

Fig.3. Metal sensor

\subsection{Humidity Sensor}

Use SHT3x temperature and humidity sensor module to communicate with STM32, and turn it on when machine vision cannot identify the garbage, so as to realize the identification of wet garbage.

The module applies digital module acquisition technology and temperature and humidity sensing technology, and the product has high reliability and long-term stability. It had the built-in humidity and temperature sensor elements, analog to digital converters, signal processing, calibration data and $\mathrm{I} 2 \mathrm{C}$ host interface. The humidity sensor is shown in Fig.4.

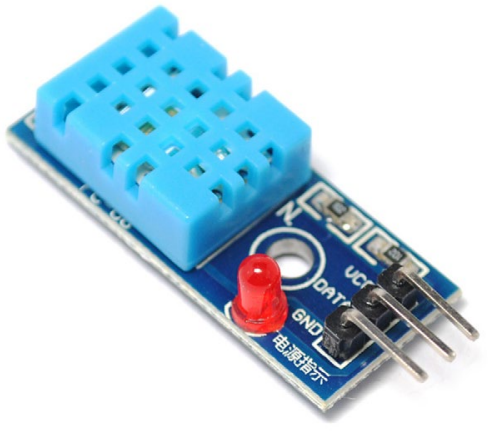

Fig.4. Humidity Sensor

\subsection{Capacitive proximity switch}

The second part of the material identification is mainly realized by the capacitive proximity switch M8M12. It can detect any dielectric substance, including conductors, semiconductors, insulators, and even can be used to detect liquid level and powdered materials. For non-metallic substances, the movement distance is determined by the dielectric constant of the material. The greater the dielectric constant of the material, the farther the sensing distance is.

The distance between it and the pallet is controlled by the motor to realize the classification of different materials. The capacitive proximity switch M8M12 is shown in Fig.5.

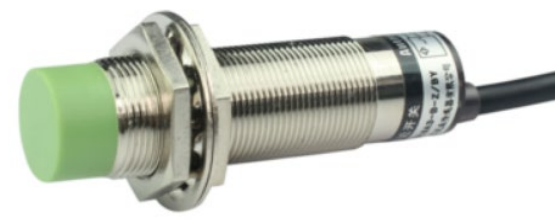

Fig.5. Capacitive proximity switch M8M12

\subsection{VOC air quality sensor}

The detection of hazardous waste that cannot be identified by machine vision is mainly achieved through the ZP16 digital VOC air quality sensor module. The air quality module uses advanced chip thick film semiconductor gas sensors. The gas sensors are resistant to formaldehyde, benzene, carbon monoxide, and ammonia, Hydrogen, alcohol, cigarette smoke, flavors and other organic volatile gases have extremely high sensitivity. After aging, debugging, calibration and adjusting, the module has good consistency and extremely high sensitivity. Extremely high sensitivity, excellent long-term stability, factory calibration, convenient and quick to use; with sensor fault self-diagnosis function, low power consumption and long life. The VOC air quality sensor is shown in Fig.6.

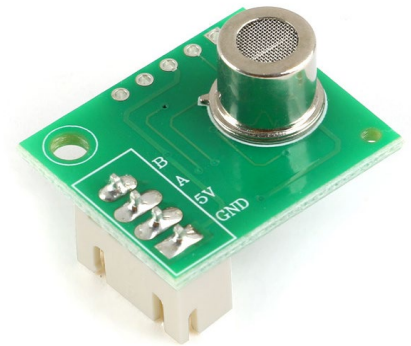

Fig.6. VOC air quality sensor

\section{OpenMV}

The vision part uses the openMV single-chip microcomputer to communicate with the STM32. The OpenMV camera is a small, low-power, low-cost circuit board that can be scripted by a high-level language Python (to be precise, MicroPython). We can use an external terminal to trigger shooting or execute an algorithm, or use the result of the algorithm to control the IO pin.

OpenMV uses STM32H743II ARM Cortex M7 processor, $480 \mathrm{MHz}, 1 \mathrm{MB}$ RAM, $2 \mathrm{MB}$ flash. All I/O pins output $3.3 \mathrm{~V}$ and are $5 \mathrm{~V}$ tolerant ${ }^{3}$. OpenMV is shown in Fig. 7. 


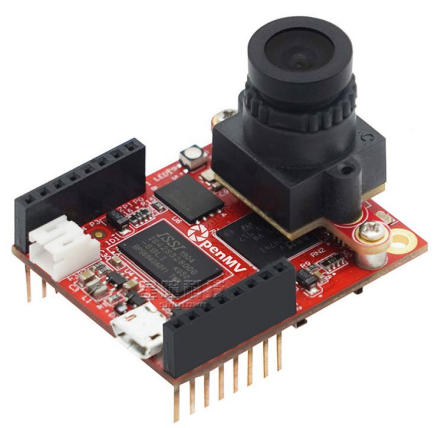

Fig.7. OpenMV

\section{Overflow Reminder Module}

The overflow detection uses the infrared distance measuring sensor module GP2Y0A21YK0F $10-80 \mathrm{~cm}$ distance sensor, the integrated combination of PSD (position sensitive detector), IRED (infrared light emitting diode) and signal processing circuit, the reflectivity of various objects, The environment temperature and working time are not easily affected by the distance detection. When the garbage overflows, the return value of the module will have a big change. When the return value meets certain conditions, the main control will transmit the corresponding information to the management terminal via the WIFI module. The overflow reminder module is shown in Fig.8.

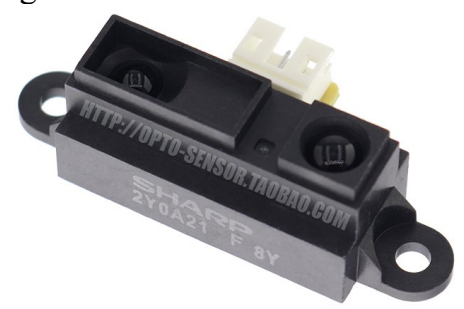

Fig.8. Overflow reminder module

\section{Conclusion}

Aiming at the problem of public garbage classification, this paper proposes a classification method based on the combination of machine vision and auxiliary sensors; designing an automatic garbage classification hardware system to realize the automatic classification and recycling of common garbage in public places in life. Tests show that the classification method is effective. The hardware and software system works stably. It shows that the broad application prospects of the research results of this paper in waste classification and environmental protection.

In the research process of this article, we found that there are some problems worthy of further digging. First, we can make greater improvements in the detection algorithm to improve the accuracy of small garbage recognition. Second, we can study the design of multi-bin garbage cans or other structures for garbage collection device to meet the sorting needs of more target categories.

\section{Acknowledgements}

The research is partly supported by the Project of Tianjin Enterprise Science and Technology Commissioner to Tianjin Tianke Intelligent and Manufacture Technology Co., Ltd (19JCTPJC53700). It is also supported by the Industry-University Cooperation and Education Project (201802286009) from Ministry of Education, China.

\section{References}

1. Golnabi, H., A. Asadpour. Design and application of industrial machine vision systems. Robotics and Computer-Integrated Manufacturing, 2007, 23(6): 630-637.

2. Liu, Jianghai, Yadong Jiang. Design of intelligent trash can be based on machine vision. 2020 International Conference on Image, Video Processing and Artificial Intelligence. Vol. 11584. International Society for Optics and Photonics, 2020.

3. Vernon, David. Machine vision-Automated visual inspection and robot vision. STIA 92 (1991): 40499. 